\title{
Numerical investigation of blanking for metal polymer sandwich sheets
}

\author{
Florian Gutknecht ${ }^{1, a}$, David Übelacker ${ }^{2}$, Till Clausmeyer ${ }^{1}$ and A. Erman Tekkaya ${ }^{1}$ \\ ${ }^{1}$ Institute of Forming Technology and Lightweight Construction (IUL), TU Dortmund, Germany \\ ${ }^{2}$ Institut für Produktionstechnik und Umformmaschinen (PtU), TU Darmstadt, Germany
}

\begin{abstract}
Metal polymer sandwich sheets consist of materials with drastically different mechanical properties. Due to this fact and because of high local gradients in the cutting zone during the blanking process, traditional process strategies and empirical knowledge are difficult to apply. A finite-element simulation of the shear cutting process is used to predict the necessary force and the geometry of the cutting surface. A fully-coupled ductile damage model is used for the description of the material behaviour. This model considers the influence of shear and compression-dominated stress states on the initiation of damage. Experimental tensile and compression test data is used for the identification of material parameters. The results of the blanking simulation are compared with experimental data. Furthermore, the evolution of the stress state is analysed to gain understanding of the underlying physics. Finally this model enables the prediction of core compression and other quantities such as the acting stresses and corresponding triaxilities, which provide valuable information for the development of analytical models.
\end{abstract}

\section{Introduction}

Sandwich sheets are excellently suited to fulfil conflicting requirements for weight reduction and increasing bearing capacity, and as a consequence replace monolithic sheets in some applications. Usually their structure consists of two thin metal sheets on the outside and a light and inexpensive core of polymer. For the sandwich investigated in this paper, the thickness and compliance is several times larger than the outer sheets. Due to the combination of materials with drastically different properties and high local gradients in the cutting zone during blanking process, traditional process strategies and empirical knowledge are no longer applicable. The selection of different materials with varying properties and thicknesses for the core, as well as the surface layers leads to a vast spectrum of possible effective behaviour, and thus offers a wide range of technological applications. But to establish this new class of material the users of this technology need to know how to process these panels. As single or multiple blanking occur in the process chain of all sheet metal parts, a physical understanding of the blanking process has high technological relevance. In particular, the knowledge of the stress state is important because it provides information on the load of tools, but is also assumed to be closely connected to the attributes of the finished product, such as the cut surface quality [2].

Even though several authors investigated the blanking of sandwich panels experimentally $[1,2,7]$, the number of studies on the simulation of the blanking process of sandwich sheets is limited. However, Chen et al. [3] have presented a numerical investigation of half-sandwichpanels with only one skin sheet.
Complementary numerical investigation is advised, as those studies can support existing theories and might give new insights, because experimental investigation cannot give any statement about stress states. Moreover, information about strains is difficult to obtain due to the small deformation zone and high local gradients. Besides experimental and numerical investigations analytical methods may be used for analysis; yet up to now they are not able to provide an adequate insight in surface quality, or force requirements. However, a first experimental observation to determine the maximum force during blanking of sandwich panels is presented by Übelacker et al. [1].

Due to the complexity of both, material and process the single layers of the panel are modelled instead of an average effective composite behaviour. Thus, this paper starts with characterization of the single layers. Afterwards the setup for shear cutting tests is presented.

In section three the numerical process model built is presented. Moreover, details on the choice of constitutive models are given.

Section four presents the surface quality and force displacement curve of different setups in experimental shear cutting. The results of a single setup are compared to the corresponding simulation results. An analysis of the stress state evolution reveals insights into the physics of shear cutting sandwich panels.

\section{Experimental investigations}

An aluminium-polymer sandwich panel, commercially available as Alucobond from 3A Composites, is examined.

${ }^{a}$ Corresponding author: Florian.Gutknecht@iul.tu-dortmund.de 
As depicted in Figure 1, the sandwich panel has a symmetrical structure.

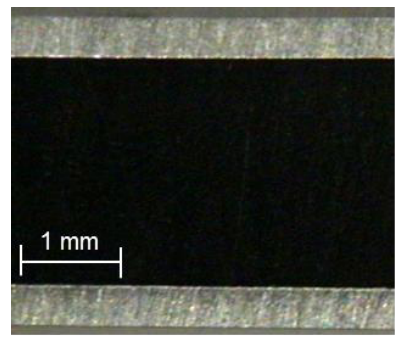

Skin sheet

LDPE core

Skin sheet

Figure 1. Microsection of the used aluminium-polymer sandwich panel.

The skin of the sandwich is manufactured from $0.5 \mathrm{~mm}$ sheets of Aluminium EN AW 5005A H22/H42 and the core is taken from a $3.3 \mathrm{~mm}$ thick panel of low density Polyethylene (LDPE). During the manufacturing process the single layers are glued together. To this end, two thin adhesive foils are placed between the skin sheets and the core material. The bonding process is initialized by high temperatures and a constant pressure. Thus the thickness of the composite is reduced from $4.3 \mathrm{~mm}$ to a final thickness of $4 \mathrm{~mm}$.

\subsection{Material characterisation}

For characterisation of the sandwich behaviour single unprocessed layers of core and skin material are delivered by the manufacturer. These layers are tested in different setups, given below. Some deviations in the mechanical behaviour of the single layers compared to the complete sandwich are expected, because the layers in the sandwich panel have already undergone some forming steps. For investigation of the elastic-plastic behaviour of the aluminium uniaxial tensile tests according to DIN 50125 are performed. Thus the specimens have been loaded at a constant strain-rate of $0.00251 / \mathrm{s}$. Modelling of damage requires the determination of fracture strains for different triaxialities [8], e.g. ratio of hydrostatic stress versus von Mises stress. Due to physical limitations of testing the 0.5 $\mathrm{mm}$ thick sheets in shear and compressive loading, fracture strains from Sun et al. [5] for a similar material are used as fracture locus.

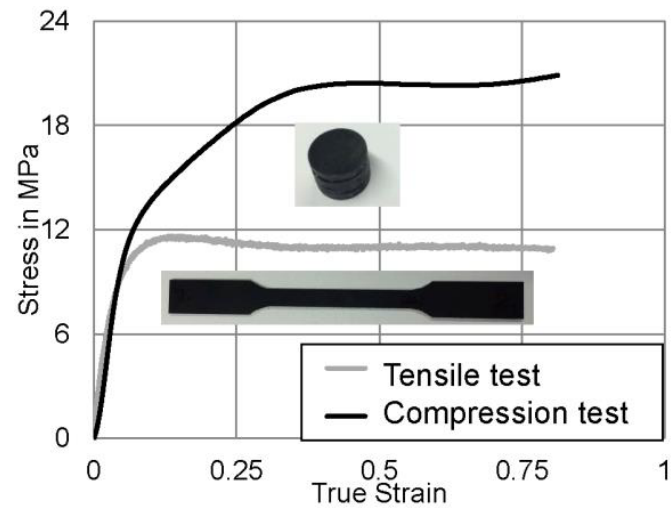

Figure 2. Polymer stress strain curve for tensile and compressive loading. [3]

For identical LDPE sheets uniaxial tensile and compressive test have been performed by Chen et al. [3] according to DIN EN ISO 527 and DIN EN ISO 604 respectively. Results are shown in Figure 2. The initial yield stress is similar in tension and compression. For larger levels of strain a substantial difference is observed.

\subsection{Blanking tests}

A conventional shear cutting tool is used for the experimental investigation. A detailed view a) and an illustration/photography b) of the tool are depicted in Figure 3. The cylindrical punch (B) is guided by a standardized guidance (C). During the downward stroke the sandwich sheet is cut along the die $(\mathrm{F})$. The sheets are positioned in a material inlet (E). During the backward stroke the sheet and the punch are separated by a stripper (D). To measure the cutting forces a Kistler 9051A force measuring ring is used (A). The displacement curve is measured by an inductive displacement transducer HBM WA/100 (G). A HBM Quantum is used as measuring amplifier.

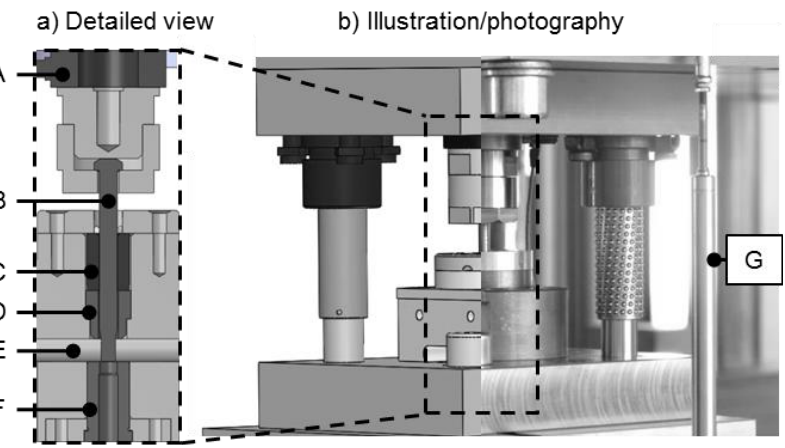

Figure 3. Experimental setup of the shear cutting tool [1].

All experiments are performed on a Synchropress SWP 2500 direct-driven servo press. The experimental parameters are listed in Table 1. As listed, a sine curve with amplitude of $25 \mathrm{~mm}$ and an average cutting speed of 50 $\mathrm{mm} / \mathrm{s}$ is used. Within the parameter variation, two different punch diameters and five clearances are investigated. A lubricant free shear cutting process is used to prevent an absorption of the lubricant by the composite.

Table 1. Experimental parameters.

\begin{tabular}{lc}
\hline Experimental parameters & \\
\hline Punch diameter $[\mathrm{mm}]$ & 8 and 32 \\
Punch and die material & $1.3343(64 \pm 2 \mathrm{HRC})$ \\
Cutting edge radius $[\mu \mathrm{m}]$ & 15 \\
Clearance $\mathrm{u}[\mu \mathrm{m}]$ & $25 / 50 / 100 / 200 / 400$ \\
Avg. Cutting Speed $[\mathrm{mm} / \mathrm{s}]$ & 50 \\
Stroke curve type & sine \\
Amplitude $[\mathrm{mm}]$ & 25 \\
\hline
\end{tabular}




\section{Simulation model}

Previous investigations [4] have shown that even conventional shear cutting of monolithic sheets poses some challenges. On the one hand the constitutive model has to account for different aspects of the material behaviour (e.g. plasticity, damage depending on the stress state). On the other hand the numerical method has to be capable to deal with severe deformation and high gradients in space and time (fast increase of plastic strain and damage in a narrow zone).

\subsection{FE-Setup}

Due to the characteristics of the process, namely strong nonlinearity of the constitutive behaviour, an explicit FEM approach is chosen. The framework presented in [3] is briefly reviewed. Assuming an additive split of the symmetric part $\mathbf{d}=\operatorname{sym}(\mathbf{l})$ of the velocity gradient $\mathbf{l}=\dot{\mathbf{F}} \cdot \mathbf{F}^{-1}$ into an elastic part $\left(\mathbf{d}^{e}\right)$ and a plastic part $\left(\mathbf{d}^{p}\right)$ $\mathbf{d}=\mathbf{d}^{e}+\mathbf{d}^{p}$, one obtains the Jaumann-type stress update

$$
T^{J}=(1-D)\left(\frac{E v}{(1+v)(1-2 v)} \operatorname{tr}\left(\mathbf{d}^{e}\right) \mathbf{1}+\frac{E v}{(1+v)} \mathbf{d}^{e}\right)
$$

for the general case of fully-coupled elasto-plasticity, with F the deformation gradient, $D$ the damage variable as well as $E$ the Young's modulus and $v$ the Poisson's ratio. $\operatorname{tr}(\bullet)$ denotes the trace operator. The general form of the yield condition for coupled damage and plasticity with isotropic hardening is

$$
\Phi=\frac{1}{(1-D)} \sigma_{e q v}-\sigma_{y}(\alpha) \leq 0
$$

where $\sigma_{y}$ and $\alpha$ represent the deformation-dependent yield stress and the equivalent plastic strain, respectively.

All simulations are performed with the software ABAQUS/explicit. Assuming isotropic material behaviour, an axisymmetric setup is chosen. The minimum element-size is set to $0.02 \mathrm{~mm}$ in a structured mesh (Figure 4).

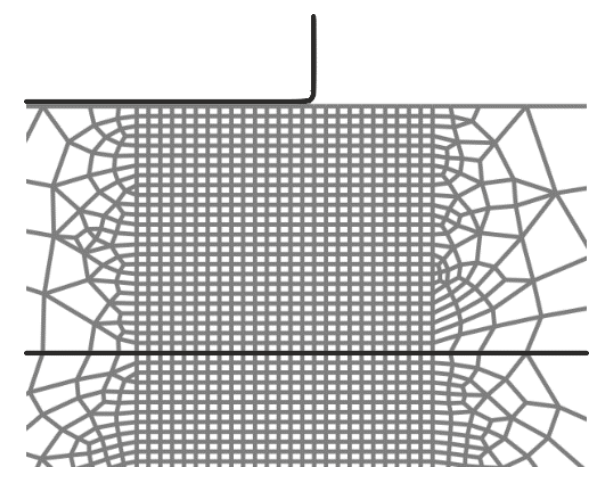

Figure 4. Detail of spatial discretization

Compared to the shear cutting of monolithic steel the usage of an Arbitrary-Lagrange-Euler (ALE) step for adjusting mesh topology is not necessarily required, as the mesh distortion is smaller compared to blanking simulation of monolithic sheet and thus omitted. Contact with the tools is considered via a kinematic formulation and a friction coefficient of 0.1 in the framework of Coulomb's model. The tools are modelled as discrete rigid bodies with an edge-radius of $0.03 \mathrm{~mm}$.

\subsection{Material model}

The heterogeneous material properties of the sandwichpanel in combination with strong local effects motivate the modelling of the individual sandwich-layers. Any softening in the individual layers is captured with the damage routines provided by ABAQUS. Initially a damage initiation criteria has to be defined. When the criteria is fulfilled, an evolution of damage variable $D$, coupled to plasticity according to equation 2, may be defined. As soon as the damage reaches a value of 1 the stiffness of the corresponding element is set to a small value and does no longer contribute to the solution. To counter the natural mesh-dependence of local damagemodels, either an energy or displacement at fracture may be defined.

\subsubsection{Aluminium}

For the aluminium the equivalent stress in equation 2 is chosen as the von Mises stress, thus $\sigma_{e q v}=\sigma_{e q v}^{v M}:=\sqrt{3 / 2}\|\operatorname{dev}(\mathbf{T})\|$. Furthermore the hardening is assumed to be isotropic. From the tensile tests the elastic parameter Young's Modulus $E$ and poison ratio $v$, as well as the ultimate tensile strength $R_{m}$ and the corresponding hardening exponent $n$ are determined. The latter two values can be used to determine the coefficients for the applied hardening law $\sigma_{y}=C \alpha^{n}$. The values are given in Table 2.

Table 2. Elastic and plastic material parameters for aluminium.

\begin{tabular}{|c|c|}
\hline $\begin{array}{c}\text { Material } \\
\text { parameter }\end{array}$ & Value \\
\hline $\begin{array}{c}\text { Young's } \\
\text { Modulus } E\end{array}$ & $75000 \mathrm{MPa}$ \\
\hline Poison ratio $v$ & 0.3 \\
\hline $\begin{array}{c}\text { Max. uniform } \\
\text { strain } n\end{array}$ & 0.037 \\
\hline $\begin{array}{c}\text { Extrapolation } \\
\text { coefficient } C\end{array}$ & $176 \mathrm{MPa}$ \\
\hline
\end{tabular}

The equivalent plastic strain at the onset of damage $\bar{\varepsilon}_{D}^{p l}$, according to [6] is assumed to be a function of stress triaxiality $\eta=\sigma_{\text {hyd }} / \sigma_{e q}$. With $\sigma_{\text {hyd }}$ and $\sigma_{e q}$ being the hydrostatic and von Mises stress respectively. At each increment during the analysis the incremental increase $\Delta \omega_{D}$ is computed.

$$
\Delta \omega_{D}=\frac{\Delta \bar{\varepsilon}^{p l}}{\bar{\varepsilon}_{D}^{p l}(\eta)} \geq 0
$$

The values of $\bar{\varepsilon}_{D}^{p l}(\eta)$ used in this analysis, can be found in [5]. For the evolution of damage a fracture energy of 10 $\mathrm{kJ}$ has been found iteratively. 


\subsubsection{Low density Polyethylene (LDPE)}

Preliminary simulations [4] revealed that during the blanking process of sandwich sheets the stress state is predominantly compressive, i.e. negative values of the hydrostatic stress are observed in the polymer. Therefore it is assumed that accurate modelling of the behaviour during compression is more important, than the prediction of the behaviour during tensile stress states, i.e. for positive values of normal stress. Consequently, a von Mises plasticity model with isotropic hardening, which is determined from compression test, is chosen for the polymer (Figure 2).

From tensile tests a failure strain of 0.4 could be determined. This value was extrapolated for other triaxialities, to obtain a function $\bar{\varepsilon}_{D}^{p l}(\eta)$ for damage initiation. The corresponding values are given in Table 3. For the evolution of damage, a displacement at fracture of 0.05 has been determined iteratively.

Table 3. Failure strain of LDPE.

\begin{tabular}{|c|c|}
\hline Triaxiality & $\begin{array}{c}\text { Failure } \\
\text { strain }\end{array}$ \\
\hline-3.0 & 1.0 \\
\hline-1.0 & 0.5 \\
\hline 0.0 & 0.4 \\
\hline 0.33 & 0.4 \\
\hline 1 & 0.1 \\
\hline
\end{tabular}

\subsubsection{Interface}

Experiments of Chen et al. [3] show that the interface under mode I loading will fail at a maximum stress of 24 MPa. Recalling that the maximum flow stress of LDPE is below $12 \mathrm{MPa}$ under tensile loading, the strength of the interface is assumed to be superior to the core material. Early simulations have shown dominance of pressure stress state in the entire process-cycle. Both arguments support the assumption that the behaviour of the interface has a small influence on the cutting force and the cutting surface. As the interface is supposed to have negligible effect on the overall performance of the shear cutting process, the constitutive behaviour of the interface is modelled by an ideal strong bonding. Therefore explicit prediction of delamination is not possible. However, the focus is on prediction of blanking force and shape deviations.

\section{Results and Discussion}

\subsection{Blanking tests}

The experimental data used for the validation of the numerical model is part of a greater experimental study presented by Stein et. al [2]. In Figure 5, microsections of the cutting surfaces are shown. As depicted, the cutting surface is strongly influenced by the cutting parameters. In general, the face bending increases with larger punch diameters. This can be explained by the acting tangential stresses in the core. In case of a smaller punch diameter the tangential stresses are higher compared to the larger punch. As a consequence, the resistance of the core material towards a deformation rises. This fact leads to a better support (i.e. transfer of axial forces) of the upper skin sheet and an earlier crack initiation.

Besides the punch diameter, the clearance between punch and die has a significant influence on the face bending. Because of the higher bending component, larger clearances lead to a higher face bending. Smaller clearances lead to parallel cutting surfaces.

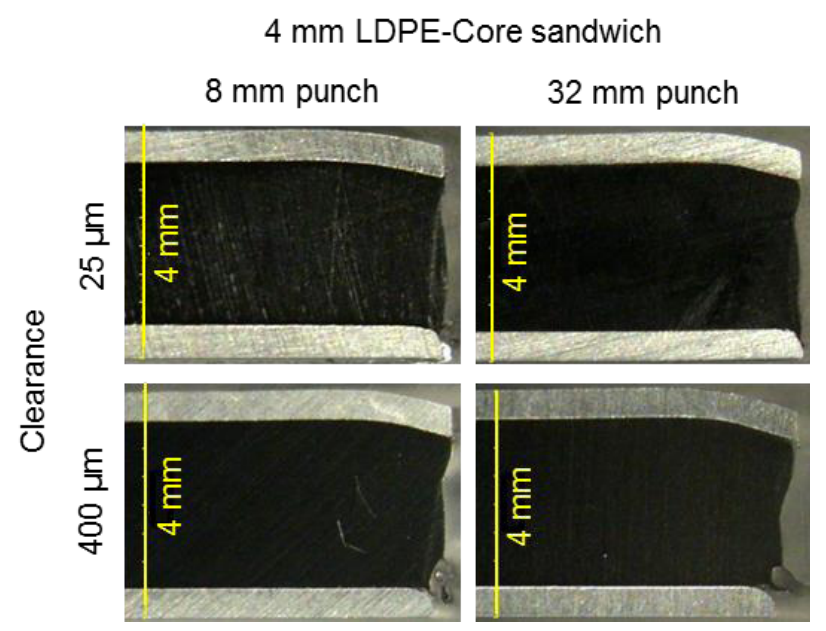

Figure 5. Cutting surfaces of LDPE-Core Sandwich [2]

In addition to the analysis of the cutting surfaces the cutting force is measured. Figure 6 shows typical force displacement curves for metal polymer sandwich sheets. The force displacement curve is separated in four main sections. The first section is dominated by a compression of the polymer core. In the second section the shearing process of the skin sheets occurs. When the skin sheets are cut, the third section begins and the core material is sheared. In the final section the force is dominated by the friction between the different layers and the shell surface of the punch. However, the clearance influences the blanked surface as discussed before. In general, a high level of parallelism caused by small clearances leads to more friction between the punch and the surface of the hole. As depicted in the force displacement curve this effect is dominant at the end of the process.

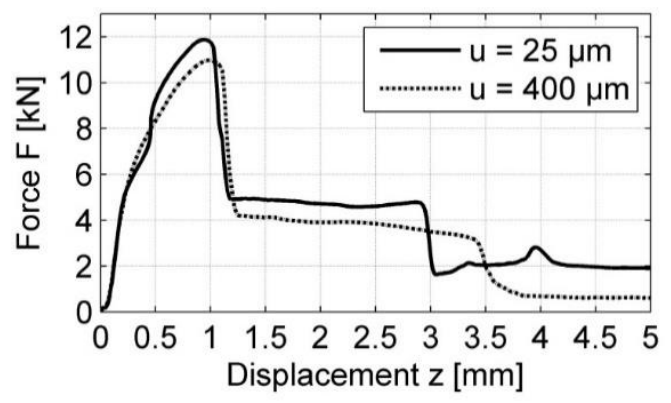

Figure 6. Force displacement curve for different clearences, $\mathrm{D}=32 \mathrm{~mm}[2]$ 
In Figure 7, a specific force displacement curve for different punch diameters is shown. As depicted a delayed rupture of the face sheets can be seen when cutting with smaller punch diameters. This can be explained by the influence of the axial stresses. In case of a smaller punch diameter the ratio of the hole area to the cutting length decreases and the axial forces increase. Therefore, the core material will be more compressed before the rupture of the lower face sheet occurs.

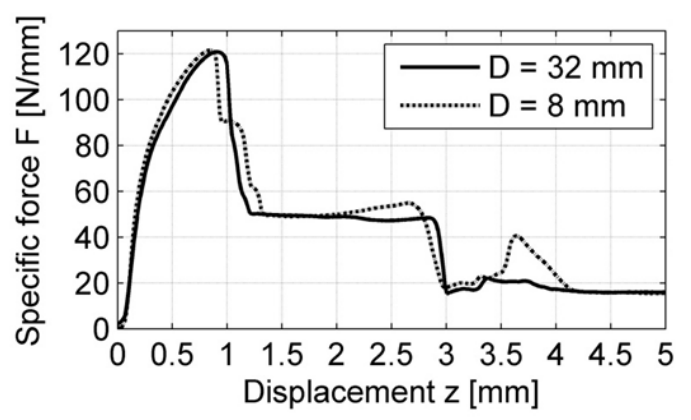

Figure 7. Specific force displacement curve for different punch diameters, $\mathrm{u}=50 \mu \mathrm{m}[2]$

\subsection{Blanking simulation}

\subsubsection{Validation}

With the setup given in previous sections, the results of the simulation are compared to the experimental ones.

For punch diameter $\mathrm{D}=8 \mathrm{~mm}$ and clearance $\mathrm{u}=100 \mu \mathrm{m}$ a face bending of $290 \mu \mathrm{m}$ could be determined (Figure 8 left). The corresponding simulation predicts a face bending of $340 \mu \mathrm{m}$ (Figure 8 right). The relative error of $17 \%$ might be improved using a finer discretization in the cutting zone, as the precise identification on severely deformed elements is difficult. Other measures for cutting surface quality, as used for monolithic sheets, such as height of burnish or fracture are not examined, as they are no longer applicable.
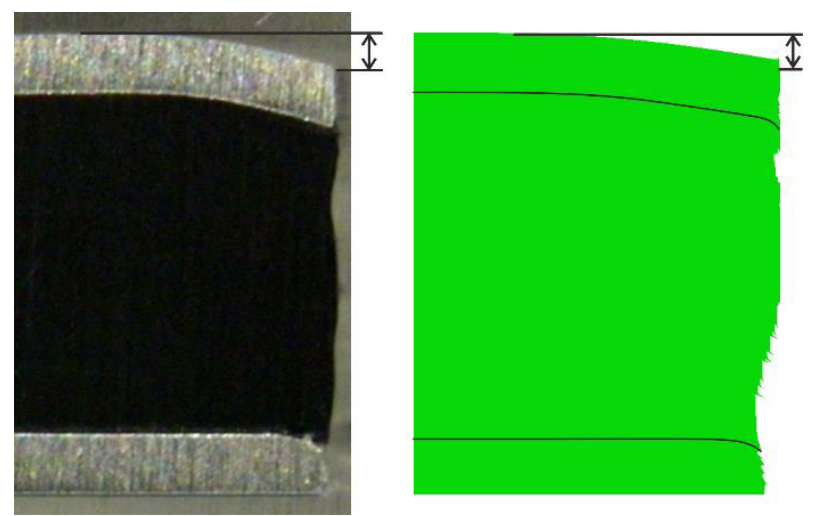

Figure 8. Cutting surfaces of LDPE-Core Sandwich from experiment (left) and simulation (right) for $\mathrm{D}=8 \mathrm{~mm}$ and $\mathrm{u}=100 \mu \mathrm{m}$

The force displacement curve is shown in Figure 9. Three distinct decreases in the force-displacement curve are identified. These are characteristic for shear cutting of sandwich panels. The first decrease indicates the rupture of the upper skin sheet, the second the separation of the lower skin sheet and the last descent occurs, when the polymer and thus the entire sandwich-sheet is separated.

While the characteristic shape of the curve for the punch force in the simulation meets the experimental very well, the maximum force has a relative error of about $20 \%$. This discrepancy is expected to improve, with a more precise parameter identification for the fracture curve of the aluminium. A tougher fracture curve would result in later separation of the skin sheets and thus improve both maximum force and punch displacement of decrease towards the values observed in experiments.

The last decrease in the force-displacement curve is determined by the constitutive behaviour of the polymer. The maximum force is reached quite satisfactorily, but decreases quasi instantly. This is a result of the pragmatic approach for the extrapolation of the fracture curve and failure energy. Both fracture strain and energy were determined with rather small values, as a much more ductile behaviour would result in numerical difficulties that are hard to taken into account, even with an ALE approach. Beside that use of ALE is questionable when multiple materials are included. Alternative approaches like remeshing result in both very small elements and wide spread of these elements due to the enormous gradients in the cutting zone. Thus the computational cost would increase enormously.

The qualitative behaviour of the blanking of the sandwich sheet is well predicted. However, the model can be improved by using more sophisticated material models for damage and failure. Due to the good qualitative agreement, further analysis of simulation results with respect to the qualitative behaviour and general physics is applicable.

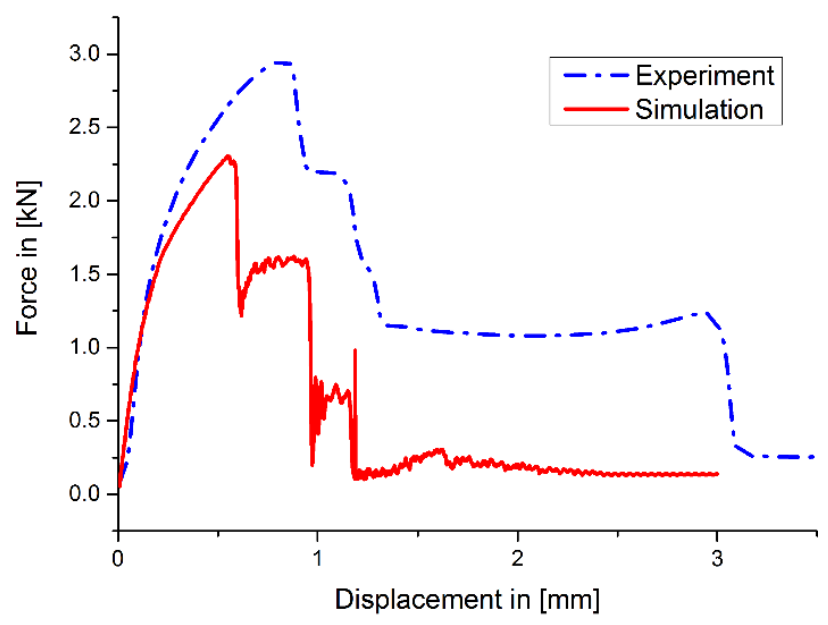

Figure 9. Force displacement curve for $\mathrm{D}=8 \mathrm{~mm}$ and $\mathrm{u}=100 \mu \mathrm{m}$

\subsubsection{Analysis}

A detailed analysis of the evolution of stress state, reveals that the physics of shear cutting sandwich-sheets differs significantly from the stress state of shear cutting monolithic sheets. The kinematics of the cutting process are described by the relative punch displacement $x=$ $x_{P} / t . x_{P}$ is the absolute displacement of the punch and $t$ the sheet thickness. Furthermore, it is known that stress 

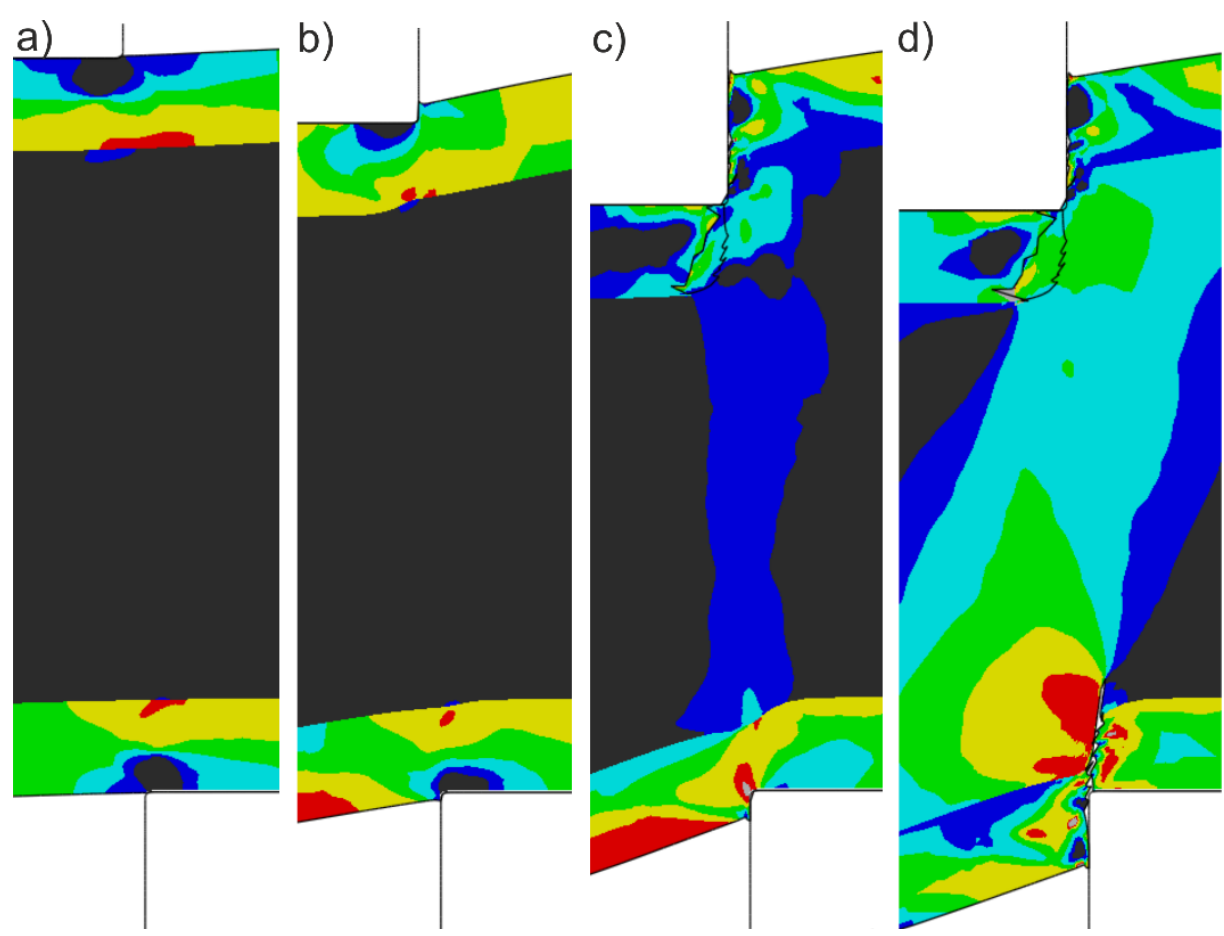

\section{Triaxiality}

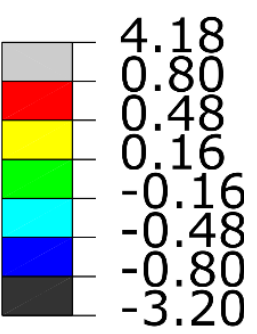

Figure 10. Distribution of triaxiality in the sandwich for relative punch-displacement a) $4 \%$, b) $14 \%$, c) $24 \%$ and d) $25 \%$

triaxiality $\eta$ is characteristic for certain states of stress. Thus $\eta=0$ corresponds to shear, $\eta= \pm 1 / 3$ is equivalent to uniaxial tension/ compression and an equi-biaxial stress state in tension/ compression corresponds to $\eta= \pm 2 / 3$. For small punch penetrations $(x \approx 4 \%)$ the triaxiality indicates a bending of the skin sheets, while the core is compressed approximately under a low triaxiality (Figure $10 \mathrm{a})$ ). This state is predominately retained up to the point before rupture of the upper skin-sheet $(x \approx 14 \%)$, with slight reduction of compressive states (Figure $10 \mathrm{~b})$ ). After the upper skin-sheet has been separated $(x \approx 24 \%)$, the triaxiality in the core changes to only biaxial compression and the lower skin sheet is loaded with pure tensile stresses (Figure $10 \mathrm{c})$ ). When both skin-sheets are separated $(x \approx$ $25 \%)$ the triaxiality in the core adopts the pattern typical for shear-cutting of monolithic sheets (Figure $10 \mathrm{~d}$ )). This pattern has been described in detail by Gutknecht et al. in [4]. Compared to the processing of monolithic sheets the following differences are found for the skin sheets:

- The loading state is comparable to a bending case.

- Significantly reduced compressive and shear stress states, thus there is a vanishing influence of those stress states in damage evolution.

- Increased occurrence of uniaxial tensile stresses.

- Damage initiation in biaxial tensile stress states.

Knowing that the failure strain for high triaxiality may be determined at moderate cost via tensile and notched tensile specimens, while failure strains for low triaxialities in thin sheets are much harder to determine, these findings are pleasant. As a consequence characterisation tests for failure strain of the skin sheets might be easier as it is sufficient to consider tests with positive triaxiality.

For investigation of axial stresses transferred by the core the stress component in axial direction is depicted in Figure 11. The analysis reveals that the zone with a minimum transferred compressive stress of $20 \mathrm{MPa}$ (maximum flow stress of LDPE) has a width of approximately $3 \mathrm{~mm}$, which is identical to the core's undeformed thickness. This zone is not symmetrical with respect to the clearance. In the skeleton this zone extends for $1 \mathrm{~mm}$ and is almost constant over the height of the core. On the other hand in the coin the stress distribution depends significantly on the height. Below the punch this zone ranges $2 \mathrm{~mm}$ into the coin and decreases to $0.3 \mathrm{~mm}$ on the opposite.

After all one should keep in mind that the constitutive behaviour of the layers, that have been characterized and used for simulation may differ from the final behaviour in the sandwich due to thermal processing and the applied plastic strain by compression during sandwich manufacturing.

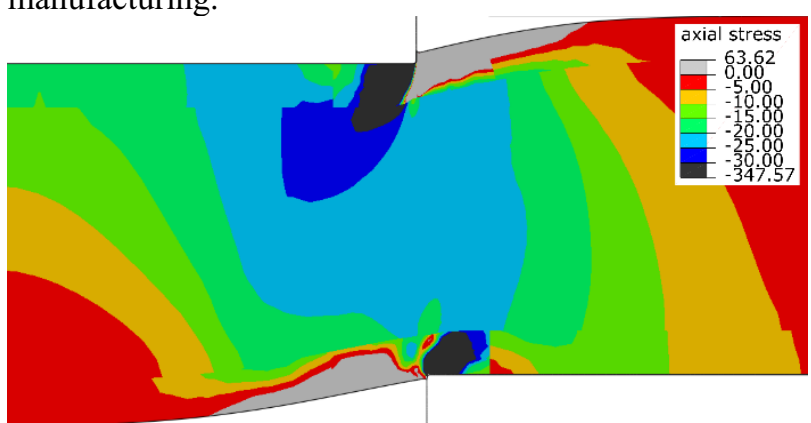

Figure 11. Distribution of axial stress in $\mathrm{MPa}$.

\section{Conclusion and outlook}

An approach on modelling sandwich panels for blanking in the framework of the Finite-Element Method is presented. Experimental data for the characterisation of raw layers are either gained from uniaxial tensile tests or taken from literature. Based on this test data, choices for the material models are made. The polymer is assumed to be linear elastic and the plasticity is accounted for with von 
Mises plasticity criterion. Furthermore, arguments are given to assume ideal bonding between each layer. An axisymmetric process model of shear cutting is built in the software ABAQUS/explicit. The process parameters are chosen to match those of physical process. Results of physical tests imply a significant effect of core compressibility on the cut-surface quality and the shape of force-displacement-curve.

Analysis of force-displacement curve and cuttingsurface shows good qualitative compliance of simulation results with physical tests. The analysis of stress state in the sandwich reveals that the physics are different from shear cutting of monolithic sheets. The stresses in the skin sheet correspond to those for bending. As a consequence the relevance of shear stresses for deformation and failure decreases, while tensile stresses become more important. Axial stresses in the core are found to be asymmetrical. Significant stresses (in the range of the yield stress of LDPE) are only transferred in a zone, which is approximately as wide, as the core's thickness.

In further investigations different punch diameters and clearances, as well as material thickness will be examined to confirm the presented findings.

As the constitutive behaviour of the core is found to be crucial for the characteristics of the shear cutting results, special emphasis should be paid on the characterization of LDPE.

\section{Acknowledgements}

The authors are grateful for the support of the German Research Foundation (DFG) for funding the projects DFG Te 508/37-1 and DFG Gr 1818/44-1. Both projects are embedded in the Research-Cluster PAK 678/0 "Dry Shear Cutting of Metal Laminated Composite Material" supported by DFG and Arbeitsgemeinschaft industrieller Forschungsvereinigungen "Otto von Guericke" e.V. (AiF).

Moreover, the authors would like to thank $3 \mathrm{~A}$ Composites for their support with Alucobond as well as all other contributors of the joint research project. Frank Steinbach (IUL, TU Dortmund) is thanked for providing the tensile data for aluminium.

\section{References}

1. D. Übelacker, J. Hohmann, P. Groche, Adv. Mat. Res. 1018, 137-144 (2014)

2. P. Stein, D. Übelacker, D. Holke, P. Groche, Mater. Sci. Forum 825-826, 433-440 (2015)

3. L. Chen, T. Clausmeyer, A.E. Tekkaya, Comput. Meth. Mater. Sci. 15, 162-168 (2015)

4. F. Gutknecht, F. Steinbach, T. Clausmeyer, A.E. Tekkaya, Proceedings of COMPLAS, 13, 170-181 (2015)

5. D.-Z. Sun, A. Ockewitz, Proceedings of the Euro LightMAT, 6ff (2013)

6. Dassault Systems, ABAQUS 6.14 Analysis User's Guide (2014)

7. M. Liewald, C. Bolay, S. Thullner, J. Manuf. Proc. 15, 364-373 (2013)
8. Y. Bao, T. Wierzbicki, Int. J. Mech. Sci. 46, 81-98 (2004) 